\title{
Compressions for Portal Vein Air Embolism Evacuation
}

\author{
Mohammed Elassa $^{\mathrm{a}, \mathrm{c}}$, Javier Gomez ${ }^{\mathrm{a}}$, Michael Grinn ${ }^{\mathrm{b}}$, Derick Christian ${ }^{\mathrm{a}}$
}

\begin{abstract}
Laparoscopic entry into the abdomen with subsequent insufflation can be achieved via an open or closed approach. A risk that is often overlooked as a result of its rarity is a venous air embolism (VAE) from carbon dioxide $\left(\mathrm{CO}_{2}\right)$ insufflation. Although both closed and open techniques of entry into the abdomen contribute to an extremely low risk for VAE, when the effects are clinically significant the mortality rate has been reported to be as high as $28 \%$. In this report we present a case of a 64-year-old female who underwent a closed approach for entry into the abdomen for elective hepatic cystectomy, who subsequently went into cardiopulmonary arrest and was found to have imaging findings suggestive of VAE localized to the portal vein. Successful management decisions presented in this case report will serve as possible options for physicians to employ when encountering a similar intraoperative complication.
\end{abstract}

Keywords: Embolism; Portal vein; Laparoscopy; Liver; Hasson; Veress

\section{Introduction}

Modern surgery has relied heavily on the use of laparoscopy both to enhance visualization as well as to decrease postoperative morbidity in patients undergoing intra-abdominal procedures. Insufflation of the abdomen is typically obtained using carbon dioxide $\left(\mathrm{CO}_{2}\right)$, in order to achieve proper visualization thus allowing for adequate maneuverability during procedures. Although rare, there is a risk for an air embolism from the insufflation process. The risk is considerable when underlying organs or vascular supplies are infiltrated during the insufflation process, most commonly with a Veress needle [1]. The pathophysiology contributing to an air embolism has potential

Manuscript submitted April 9, 2021, accepted June 14, 2021

Published online June 28, 2021

aDepartment of Surgery, St. Joseph's University Medical Center, Paterson, NJ, USA

bDepartment of Anesthesiology, St. Joseph's University Medical Center, Paterson, NJ, USA

${ }^{\mathrm{c} C}$ Corresponding Author: Mohammed Elassa, Department of General Surgery, St. Joseph's University Medical Center, 703 Main St, Paterson, NJ 07503, USA. Email: mohammed.h.elassa@gmail.com

doi: https://doi.org/10.14740/jcs436 to hold severe consequences as it has the capability of impeding right ventricular outflow to the point where cardiopulmonary arrest can occur. A mortality rate as high as $28 \%$ has been reported with $\mathrm{CO}_{2}$ embolism [2]. Here we describe a case where upon closed entry into the abdomen via Veress needle, there was inadvertent intraparenchymal insufflation which led to cardiopulmonary arrest with successful subsequent management and ultimately stabilization of our patient.

\section{Case Report}

Our patient is a 64-year-old female with pertinent past medical history of right lobe hepatic cyst and past surgical history of cholecystectomy who presented to surgery clinic for elective resection of her liver cyst. She was initially diagnosed with this massive cyst 5 years prior, where she had a computed tomography scan of her abdomen and pelvis after she had presented with intractable nausea and vomiting, and was ultimately found to have gastroenteritis (Fig. 1a, b).

The patient was scheduled for diagnostic laparoscopy, resection of liver cyst and intraoperative ultrasound. Discussion with the anesthetic team and the surgeons noted that prior to surgery, two large-bore intravenous catheter (IVs) and a post induction arterial line would be appropriate for the case. In the operating room a closed technique was used to enter the abdomen. Two attempts were made to enter the abdominal cavity using a Veress needle at Palmer's point. Both attempts at entry into the abdomen demonstrated elevated cavitary pressure. During the first attempt for entry into the abdomen a pressure of $15 \mathrm{~mm}$ $\mathrm{Hg}$ was noted, Veress needle was subsequently removed and an insertion site lateral to the initial point was used. During this second attempt at entry, a pressure of $54 \mathrm{~mm} \mathrm{Hg}$ was noted. At this point the anesthesiologist in the operating room notified the surgical team that the patient became hypotensive, tachycardic with end-tidal $\mathrm{CO}_{2}$ rapidly diminished from baseline of $35 \mathrm{~mm} \mathrm{Hg}$ to $7 \mathrm{~mm} \mathrm{Hg}$. Once the anesthesiologist noticed the change within seconds after insufflation, multiple events took place simultaneously. Assistance from anesthesia team was called for, manual ventilation confirmed endotracheal intubation with repeat direct laryngoscopy. Due to significant hypotension, epinephrine 500 $\mu \mathrm{g}$ was administered as a bolus, and chest compressions were initiated by the surgical team for one round. The patient's pulse was palpable and blood pressure was noted to improve towards baseline within $90 \mathrm{~s}$. Once the patient was hemodynamically stable central venous access was achieved with a triple-lumen catheter (TLC) in the right internal jugular vein. After placement, air was noted to be mixed with blood upon aspiration. 

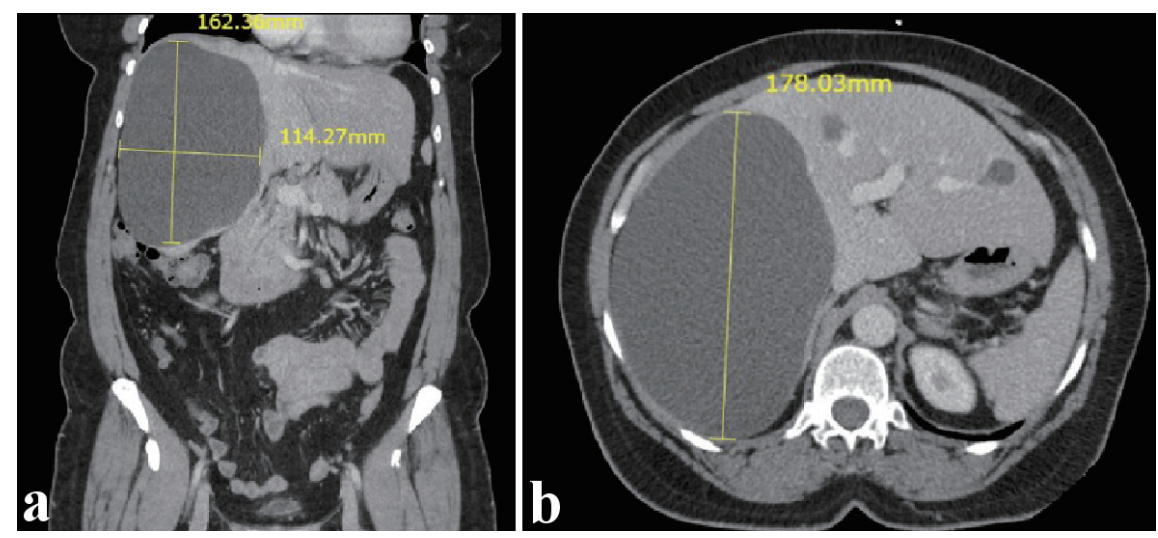

Figure 1. Computed tomography scan showing right hepatic cyst. (a) Coronal view. (b) Transverse view.

Subsequently, transesophageal echocardiography (TEE) was used for confirmation of the TLC placement. Intraoperative TEE demonstrated air in the portal vein, in the midesophageal bicaval view it showed air jetting in from the inferior vena cava, and in the midesophageal four-chamber view there was air noted in the right atrium and ventricle (Fig. 2a-c). No patent foramen ovale (PFO) was noted.

Following stabilization, there was diffusion of the embolism from the portal system and the heart that by the time the TEE was completed no more air was present in the system. The patient was extubated with no neurological deficits and transported to the Surgical Intensive Care Unit where she was evaluated and managed for hemodynamic and respiratory stability. A follow-up computed tomography of abdomen and pelvis was ordered which showed some residual air within the liver parenchyma, and minimal amount of air within the major vascular structures within the abdomen or pelvis (Fig. 3a, b).

The patient was observed, evaluated and medically optimized until she was discharged a few days postoperatively with instructions to report back the upcoming week for elective resection of the right hepatic cyst. In this final operation, the patient tolerated diagnostic laparoscopy, segment 6/7 cystectomy as well as fulguration of cyst wall. In this second attempt at cyst resection, as opposed to the initial decision of insufflating the abdomen via entry into the abdomen at Palmer's point, a supraumbilical incision was made, using Hasan technique to enter the abdomen. Entry pressures were noted to be 2 , following which appropriate pneumoinsufflation pressures were noted. Careful dissection using Ligasure was employed to perform the cystectomy. Cyst fluid was aspirated, and cyst wall was sent to for pathologic analysis which found solitary unilocular cyst compromising of single layer of cuboidal biliary type epithelium. Following full resection of the cyst, the argon beam was used to coagulate the cyst wall that remained. Our patient was extubated, recovered well in the postoperative care unit and was discharged that same day.

\section{Discussion}

Although a very rare occurrence, venous air embolism from laparoscopic insufflation can have devastating outcomes. Multiple case reports have shown similar events due to hepatic anatomical differences. The main underlying principle is rapid identification of potential life-threatening differentials. Possible differentials of anaphylaxis, extubation, kinked endotracheal tube, an obstructive process, or spontaneous pulmonary embolism (PE), should be considered. A theoretical dose of $100 \mathrm{~mL}$ of air has the capability to fill the chambers of the right heart, which can quickly lead to fatal cardiac arrest. A higher suspicion and vigilance should be set in a protocolized fashion to avoid negative outcomes. We would argue for all hepatic cases to have a protocolized approach in regard to communication between anesthesia and surgery, in regard to surgical plan, perioperative access and management. Venous and arterial access prior to surgery for the potential mitigation of treatment of unexpected complications should be protocolized, as patients with aberrant intraabdominal pathology can have significant negative outcomes. An algorithmic approach to hepatic surgery can help reduce the effects of negative outcomes.
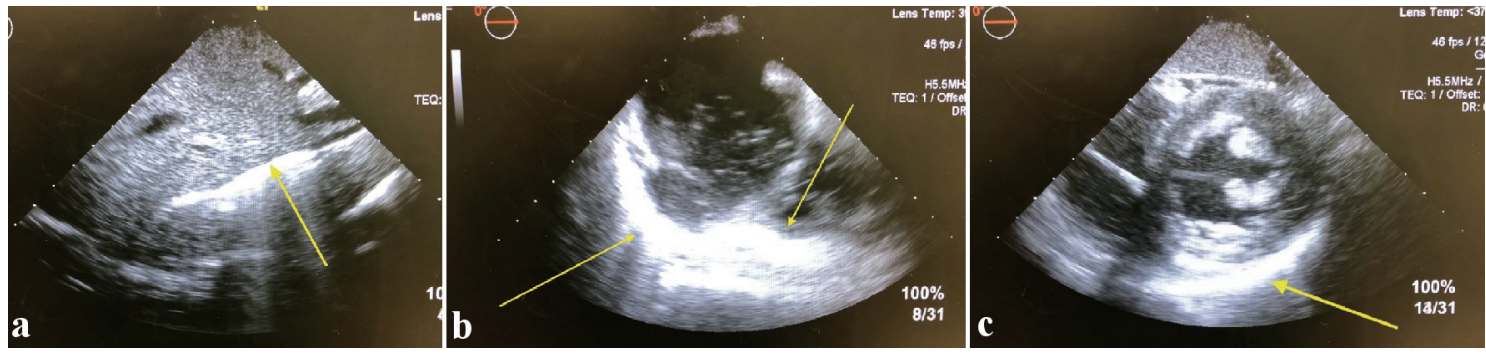

Figure 2. Transesophageal echocardiography showing findings suggestive of portal vein embolism. 


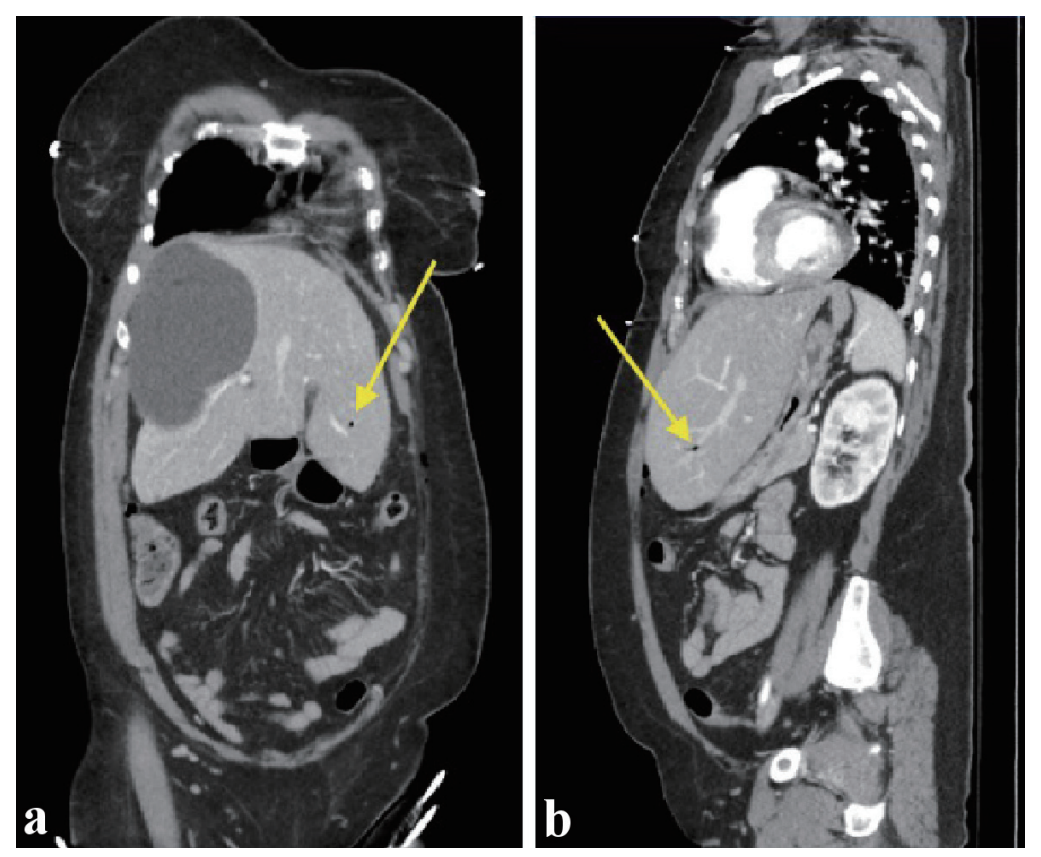

Figure 3. Computed tomography of chest, abdomen, and pelvis showing air within the hepatic vasculature. (a) Coronal view. (b) Sagittal view.

\section{Incidence}

$\mathrm{CO}_{2}$ embolism is a rare complication of laparoscopic procedures. In a meta-analysis conducted in 1997, nearly 500,000 closed entry laparoscopic procedures were studied, out of which there was a reported seven cases $(0.001 \%)$ of $\mathrm{CO}_{2}$ emboli. Another report found the incidence of $\mathrm{CO}_{2}$ emboli specifically within laparoscopic liver biopsy cases to be one in approximately $64,000(0.0016 \%)$ cases [3]. As previously explained, the incidence is extremely low; however when a patient develops a clinically significant venous air embolism there is potential for a fatal outcome, with a reported mortality rate as high as $28 \%$ [2].

\section{Clinical features}

Clinical features that suggest a $\mathrm{CO}_{2}$ embolism are all derived from the ultimate physiologic effect of right ventricular outflow obstruction. Common symptoms that should raise suspicion for an air embolus is right and left heart strain or failure, arrhythmia/ electrocardiogram (EKG) abnormalities, signs of pulmonary hypertension, systemic hypotension, "mill-wheel" murmur, decreased end-tidal $\mathrm{CO}_{2}$ and possible cardiovascular collapse [1].

\section{Diagnosis}

Intraoperative options for detection of $\mathrm{CO}_{2}$ embolism includes visual detection of air embolism or recognition of the physiological changes associated with an embolization. The most common physiological changes noted in cases with air embolism have been tachycardia, cardiac arrhythmias, hypotension, and rapid desaturation [4]. Both end-tidal $\mathrm{CO}_{2}$ monitoring as well as transesophageal Doppler have been used for detection of a $\mathrm{CO}_{2}$ embolism [5]. Specifically, a rapid decrease in endtidal $\mathrm{CO}_{2}$ is an appropriate indicator of a $\mathrm{CO}_{2}$ embolism. A study comparing the sensitivity for embolism using end-tidal $\mathrm{CO}_{2}$ monitoring and transesophageal Doppler was conducted on 10 pigs who were administered a successively larger intravenous $\mathrm{CO}_{2}$ bolus. This study showed that the transesophageal Doppler was more sensitive with smaller concentrations of $\mathrm{CO}_{2}$, with comparable sensitivity at larger concentrations.

\section{Management}

The general goals of management for a patient with suspected $\mathrm{CO}_{2}$ embolus is to initially prevent further $\mathrm{CO}_{2}$ entry, followed by reduction in the volume of the embolism while at the same time providing hemodynamic support [6]. To help prevent further $\mathrm{CO}_{2}$ entry, it is imperative to immediately discontinue insufflation and place patient in Durant's position. Durant's position requires the patient to be placed in left lateral decubitus and Trendelenburg position. The purpose of this positioning is to help facilitate the passage of the air embolism away from the right ventricular outflow tract and into the right atrium instead, in order to avoid cardiopulmonary arrest [7]. An increase in the respiratory rate and tidal volume, with maintained positive end-expiratory pressure should also be initiated to minimize any gas entrapment and increase the gradient for evacuation of $\mathrm{CO}_{2}$ [8]. In patients who have central venous access, aspiration has been reported to be successful in evacuation of the air embolism [7-9]. Theoretically once patient is in Durant's position, aspiration using central venous access would have an increased likelihood of successful evacuation of the air embolus. 


\section{Conclusions}

In summary, we present a case of a $\mathrm{CO}_{2}$ embolism during a laparoscopic cystectomy where insufflation was attempted via a closed approach. Our report serves as a reminder that this potentially fatal complication can occur however, there are management decisions which may decrease the risk for adverse outcomes. Specifically, insufflation should be stopped immediately, the patient should be placed in Durant's position, and an increase in tidal volume as well as respiratory rate should be adjusted on mechanical ventilation. Lastly, if possible, an attempt at aspiration of the embolus should be attempted.

\section{Acknowledgments}

We would like to acknowledge M.C. for allowing us to share her story.

\section{Financial Disclosure}

None to declare.

\section{Conflict of Interest}

None to declare.

\section{Informed Consent}

Not applicable.

\section{Author Contributions}

ME and MG: report writing and editing, JG and DC: report editing

\section{Data Availability}

Any inquiries regarding supporting data availability of this study should be direct to the corresponding author.

\section{References}

1. Park EY, Kwon JY, Kim KJ. Carbon dioxide embolism during laparoscopic surgery. Yonsei Med J. 2012;53(3):459-466.

2. Cottin V, Delafosse B, Viale JP. Gas embolism during laparoscopy: a report of seven cases in patients with previous abdominal surgical history. Surg Endosc. 1996;10(2):166169.

3. Bruhl W. Complications of laparoscopy and liver biopsy under vision; the results of a survey. Ger Med Mon. 1967;12(1):31-32.

4. Cadis AS, Velasquez CD, Brauer M, Hoak B. Intraoperative management of a carbon dioxide embolus in the setting of laparoscopic cholecystectomy for a patient with primary biliary cirrhosis: A case report. Int J Surg Case Rep. 2014;5(11):833-835.

5. Hong JY, Kim WO, Kil HK. Detection of subclinical $\mathrm{CO} 2$ embolism by transesophageal echocardiography during laparoscopic radical prostatectomy. Urology. 2010;75(3):581-584.

6. Mirski MA, Lele AV, Fitzsimmons L, Toung TJ. Diagnosis and treatment of vascular air embolism. Anesthesiology. 2007;106(1):164-177.

7. McCarthy CJ, Behravesh S, Naidu SG, Oklu R. Air embolism: practical tips for prevention and treatment. J Clin Med. 2016;5(11):93.

8. Smith HJ. Carbon dioxide embolism during pneumoperitoneum for laparoscopic surgery: a case report. AANA J. 2011;79(5):371-373.

9. Her AY, Kim YH, Moon DH, Kim JH, Jeong JH, Park SH, Jeong JS. Successful treatment of intracardiac air embolism using intracardiac catheter aspiration. J Geriatr Cardiol. 2017;14(2):151-152. 